\title{
The Quantum Theory of Optical Coherence*
}

\author{
Roy J. Glauber \\ Lyman Laboratory of Physics, Harvard University, Cambridge, Massachusetts
}

(Received 11 February 1963)

\begin{abstract}
The concept of coherence which has conventionally been used in optics is found to be inadequate to the needs of recently opened areas of experiment. To provide a fuller discussion of coherence, a succession of correlation functions for the complex field strengths is defined. The $n$th order function expresses the correlation of values of the fields at $2 n$ different points of space and time. Certain values of these functions are measurable by means of $n$-fold delayed coincidence detection of photons. A fully coherent field is defined as one whose correlation functions satisfy an infinite succession of stated conditions. Various orders of incomplete coherence are distinguished, according to the number of coherence conditions actually satisfied. It is noted that the fields historically described as coherent in optics have only first-order coherence. On the other hand, the existence, in principle, of fields coherent to all orders is shown both in quantum theory and classical theory. The methods used in these discussions apply to fields of arbitrary time dependence. It is shown, as a result, that coherence does not require monochromaticity. Coherent fields can be generated with arbitrary spectra.
\end{abstract}

\section{INTRODUCTION}

$\mathrm{C}$ ORRELATION, it has long been recognized, plays a fundamental role in the concept of optical coherence. Techniques for both the generation and detection of various types of correlations in optical fields have advanced rapidly in recent years. The development of the optical maser, in particular, has led to the generation of fields with a range of correlation unprecedented at optical frequencies. The use of techniques of coincidence detection of photons ${ }^{1,2}$ has, in the same period, shown the existence of unanticipated correlations in the arrival times of light quanta. The new approaches to optics, which such developments will allow us to explore, suggest the need for a fundamental discussion of the meaning of coherence.

The present paper, which is the first of a series on fundamental problems of optics, is devoted largely to defining the concept of coherence. We do this by constructing a sequence of correlation functions for the field vectors, and by discussing the consequences of certain assumptions about their properties. The definition of coherence which we reach differs from earlier ones in several significant ways. The most important difference, perhaps, is that complete coherence, as we define it, requires that the field correlation functions satisfy an infinite succession of coherence conditions. We are led then to distinguish among various orders of incomplete coherence, according to the number of conditions satisfied. The fields traditionally described as coherent in optics are shown to have only first-order coherence. The fields generated by the optical maser, on the other hand, may have a considerably higher order of coherence. A further difference between our approach and previous ones is that it is constructed to apply to fields of arbitrary time dependence, rather than just to those which are, on the average, stationary in time. We

* Supported in part by the U. S. Air Force Office of Scientific Research.

${ }^{1}$ R. Hanbury Brown and R. Q. Twiss, Nature 177, 27 (1956); Proc. Roy. Soc. (London) A242, 300 (1957); A243, 291 (1957).

${ }^{2}$ G. A. Rebka and R. V. Pound, Nature 180, 1035 (1957). have also attempted to develop the discussion in a fully quantum theoretical way.

It would hardly seem that any justification is necessary for discussing the theory of light quanta in quantum theoretical terms. Yet, as we all know, the successes of classical theory in dealing with optical experiments have been so great that we feel no hesitation in introducing optics as a sophomore course. The quantum theory, in other words, has had only a fraction of the influence upon optics that optics has historically had upon quantum theory. The explanation, no doubt, lies in the fact that optical experiments to date have paid very little attention to individual photons. To the extent that observations in optics have been confined to the measurement of ordinary light intensities, it is not surprising that classical theory has offered simple and essentially correct insights.

Experiments such as those on quantum correlations suggest, on the other hand, the growing importance of studies of photon statistics. Such studies lie largely outside the grasp of classical theory. To observe that the quantum theory is fundamentally necessary to the treatment of these problems is not to say that the semiclassical approach always yields incorrect results. On the contrary, correct answers to certain classes of problems of photon statistics ${ }^{3}$ may be found through adaptations of classical methods. There are, however, distinct virtues to knowing where such methods succeed and where they do not. For that reason, as well as for its intrinsic interest, we shall formulate the theory in quantum theoretical terms from the outset. Quite a few of our arguments can easily be paraphrased in classical terms. Several seem to be new in the context of classical theory.

We shall try to construct this paper so that it can be followed with little more than a knowledge of elementary quantum mechanics. Since its subject matter is, in the deepest sense, quantum electrodynamics, we begin with

\footnotetext{
${ }^{3}$ E. M. Purcell, Nature 178, 1449 (1956).
} 
a section which describes the few simple aspects of that subject which are referred to later.

\section{ELEMENTS OF FIELD THEORY}

The observable quantities of the electromagnetic field will be taken to be the electric and magnetic fields which are represented by a pair of Hermitian operators, $\mathbf{E}(\mathbf{r} t)$ and $\mathbf{B}(\mathbf{r} t)$. The state of the field will be described by means of a state vector, |\rangle , on which the fields operate from the left, or by means of its adjoint, \langle| , on which they operate from the right. Since we shall use the Heisenberg representation, the choice of a fixed state vector specifies the properties of the field at all times. The theory is constructed, by whatever formal means, so that in a vacuum the field operators $\mathbf{E}(\mathbf{r} t)$ and $\mathbf{B}(\mathbf{r} t)$ satisfy the Maxwell equations

$$
\begin{aligned}
\nabla \cdot \mathbf{E} & =0 \\
\nabla \times \mathbf{E} & =-\frac{1}{c} \frac{\partial \mathbf{B}}{\partial t}, \\
\nabla \times \mathbf{B} & =-\frac{1}{c} \frac{\partial \mathbf{E}}{\partial t} \\
\nabla \cdot \mathbf{B} & =0 .
\end{aligned}
$$

We omit the source terms in the equations since, for the present, we are more interested in the fields themselves than the explicit way in which they are generated or detected. It follows from the Maxwell equations that the electric field operator obeys the wave equation

$$
\left(\nabla^{2}-\frac{1}{c^{2}} \frac{\partial^{2}}{\partial t^{2}}\right) \mathbf{E}(\mathbf{r} t)=0
$$

and the magnetic field operator does likewise.

One of the essential respects in which quantum field theory differs from classical theory is that two values of the field operators taken at different space-time points do not, in general, commute with one another. The components of the electric field, which is the only field we shall discuss at length, obey a commutation relation of the general form

$$
\left[E_{\mu}(\mathbf{r} t), E_{\nu}\left(\mathbf{r}^{\prime} t^{\prime}\right)\right]=D_{\mu \nu}\left(\mathbf{r}-\mathbf{r}^{\prime}, t-t^{\prime}\right) .
$$

That the tensor function $D_{\mu \nu}$ has as arguments the coordinate differences $\mathbf{r}-\mathbf{r}^{\prime}$ and $t-t^{\prime}$ follows from the invariance of the theory under translations in space and time. We shall not need any further details of the function $D_{\mu \nu}$, but may mention that it vanishes when the four-vector $\left(\mathbf{r}-\mathbf{r}^{\prime}, t-t^{\prime}\right)$ lies outside the light cone, i.e., for $\left(\mathbf{r}-\mathbf{r}^{\prime}\right)^{2}>c^{2}\left(t-t^{\prime}\right)^{2}$. The vanishing of the commutator, for points with spacelike separations, corresponds to the fact that measurements of the stated field components at such points can be carried out to arbitrary accuracy. Such accuracy is attainable since no dis- turbances can propagate through the field rapidly enough to reach one point from the other.

An important element of the discussion in this paper will be the separation of the electric field operator $\mathbf{E}(\mathbf{r} t)$ into its positive and negative frequency parts. The separation is most easily accomplished when the time dependence of the operator is represented by a Fourier integral. If, for example, the field operator has a representation

$$
\mathbf{E}(\mathbf{r} t)=\int_{-\infty}^{\infty} \mathfrak{e}(\omega, \mathbf{r}) e^{-i \omega t} d \omega
$$

where the Hermitian property is secured by the relation $\mathfrak{e}(-\omega, \mathbf{r})=\mathfrak{e}^{\dagger}(\omega, \mathbf{r})$, then we define the positive frequency part of $\mathbf{E}$ as

$$
\mathbf{E}^{(+)}(\mathbf{r} t)=\int_{0}^{\infty} \mathfrak{e}(\omega, \mathbf{r}) e^{-i \omega t} d \omega,
$$

and the negative frequency part as

$$
\begin{aligned}
\mathbf{E}^{(-)}(\mathbf{r} t) & =\int_{-\infty}^{0} \mathfrak{e}(\omega, \mathbf{r}) e^{-i \omega t} d \omega, \\
& =\int_{0}^{\infty} \mathfrak{e}^{\dagger}(\omega, \mathbf{r}) e^{-i \omega t} d \omega .
\end{aligned}
$$

It is evident from these definitions that the field is the sum of its positive and negative frequency parts,

$$
\mathbf{E}(\mathbf{r} t)=\mathbf{E}^{(+)}(\mathbf{r} t)+\mathbf{E}^{(-)}(\mathbf{r} t) .
$$

The two parts, regarded separately, are not Hermitian operators; the fields they represent are intrinsically complex, and mutually adjoint,

$$
\mathbf{E}^{(-)}(\mathbf{r} t)=\mathbf{E}^{(+) \dagger}(\mathbf{r} t) \text {. }
$$

In the absence of a Fourier integral representation of $\mathbf{E}(\mathbf{r} t)$, the positive and negative frequency parts of the field may be defined more formally as the limits of the integrals,

$$
\begin{aligned}
& \mathbf{E}^{(+)}(\mathbf{r} t)=\lim _{\eta \rightarrow+0} \frac{1}{2 \pi i} \int_{-\infty}^{\infty} \frac{\mathbf{E}(\mathbf{r}, t-\tau)}{\tau-i \eta} d \tau, \\
& \mathbf{E}^{(-)}(\mathbf{r} t)=-\lim _{\eta \rightarrow+0} \frac{1}{2 \pi i} \int_{-\infty}^{\infty} \frac{\mathbf{E}(\mathbf{r}, t-\tau)}{\tau+i \eta} d \tau .
\end{aligned}
$$

It follows from the intrinsically different time dependences of $\mathbf{E}^{(+)}(\mathbf{r} t)$ and $\mathbf{E}^{(-)}(\mathbf{r} t)$ that they act to change the state of the field in altogether different ways, one associated with photon absorption, the other with photon emission. In particular, the positive frequency part, $\mathbf{E}^{(+)}(\mathbf{r} t)$, may be $\operatorname{shown}^{4}$ to be a photon annihilation operator. Applied to an $n$-photon state it produces an $(n-1)$-photon state. Further applications of $\mathbf{E}^{(+)}(\mathbf{r} t)$

${ }^{4}$ See, for example, P. A. M. Dirac, The Principles of Quantum Mechanics (Oxford University Press, New York, 1947), 3rd ed., pp. 239-242. 
reduce the number of photons present still further, but the regression must end with the state in which the field is empty of all photons. It is part of the definition of this state, which we represent as $|\mathrm{vac}\rangle$, that

$$
\mathbf{E}^{(+)}(\mathbf{r} t)|\mathrm{vac}\rangle=0 .
$$

The adjoint relation is

$$
\langle\operatorname{vac}| \mathbf{E}^{(-)}(\mathbf{r} t)=0 .
$$

Since the operator $\mathbf{E}^{(+)}(\mathbf{r} t)$, annihilates photons, its Hermitian adjoint, $\mathbf{E}^{(-)}(\mathbf{r} t)$, must create them; applied to an $n$-photon state it produces an $(n+1)$-photon state. In particular, the state

$$
\mathbf{E}^{(-)}(\mathbf{r} t)|\mathrm{vac}\rangle
$$

is a one-photon state.

It has become customary, in discussions of classical theory, to regard the electric field $\mathbf{E}(\mathbf{r} t)$ as the quantity one measures experimentally, and to think of the complex fields $\mathbf{E}^{( \pm)}(\mathbf{r} t)$ as convenient, but fictitious, mathematical constructions. Such an attitude can only be held be held in the classical domain, where quantum phenomena play no essential role. The frequency $\omega$ of a classical field must be so low that the quantum energy $\hbar \omega$ is negligible. In such a case, we can not tell whether a classical test charge emits or absorbs quanta. In measuring a classical field strength, $\mathbf{E}(\mathbf{r} t)$, we implicitly sum the effects of photon absorption and emission which are described individually by the fields $\mathbf{E}^{(+)}(\mathbf{r} t)$ and $\mathbf{E}^{(-)}(\mathbf{r} t)$.

Where quantum phenomena are important the situation is usually quite different. Experiments which detect photons ordinarily do so by absorbing them in one or another way. The use of any absorption process, such as photoionization, means in effect that the field we are measuring is the one associated with photon annihilation, the complex field $\mathbf{E}^{(+)}(\mathbf{r} t)$. We need not discuss the details of the photoabsorption process to find the appropriate matrix element of the field operator. If the field makes a transition from the initial state $|i\rangle$ to a final state $|f\rangle$ in which one photon, polarized in the $\mu$ direction, has been absorbed, the matrix element takes the form

$$
\left\langle f\left|E_{\mu}{ }^{(+)}(\mathbf{r} t)\right| i\right\rangle .
$$

We shall define an ideal photon detector as a system of negligible size (e.g., of atomic or subatomic dimensions) which has a frequency-independent photoabsorption probability. The advantage of imagining such a detector, as we shall show more explicitly in a later paper, is that the rate at which it records photons is proportional to the sum over all final states $|f\rangle$ of the squared absolute values of the matrix elements (2.14). In other words, the probability per unit time that a photon be absorbed by an ideal detector at point $\mathbf{r}$ at time $t$ is proportional to

$$
\begin{aligned}
\sum_{f} \mid\langle f| E_{\mu}{ }^{(+)} & \left.(\mathbf{r} t)|i\rangle\right|^{2} \\
& =\sum_{f}\left\langle i\left|E_{\mu}{ }^{(-)}(\mathbf{r} t)\right| f\right\rangle\left\langle f\left|E_{\mu}{ }^{(+)}(\mathbf{r} t)\right| i\right\rangle \\
& =\langle i| E_{\mu}\left({ }^{(-)}(\mathbf{r} t) E_{\mu}{ }^{(+)}(\mathbf{r} t)|i\rangle .\right.
\end{aligned}
$$

We may verify immediately from (2.12) that the rate at which photons are detected in the empty, or vacuum, state vanishes.

The photodetector we have described is the quantummechanical analog of what, in classical experiments, has been called a square-law detector. It is important to bear in mind that such a detector for quanta measures the average value of the product $E_{\mu}{ }^{(-)} E_{\mu}{ }^{(+)}$, and not that of the square of the real field $E_{\mu}(\mathbf{r} t)$. Indeed, it is easily seen from the foregoing work that the average value of $E_{\mu}{ }^{2}(\mathbf{r} t)$ does not vanish in the vacuum state;

$$
\left\langle\operatorname{vac}\left|E_{\mu}{ }^{2}(\mathbf{r} t)\right| \mathrm{vac}\right\rangle>0 \text {. }
$$

The electric field in the vacuum undergoes zero-point oscillations which, in the correctly formulated theory, have nothing to do with the detection of photons.

Recording photon intensities with a single detector does not exhaust the measurements we can make upon the field, though it does characterize, in principle, virtually all the classic experiments of optics. A second type of measurement we may make consists of the use of two detectors situated at different points $\mathbf{r}$ and $\mathbf{r}^{\prime}$ to detect photon coincidences or, more generally, delayed coincidences. The field matrix element for such transitions takes the form

$$
\left\langle f\left|E_{\mu}^{(+)}\left(\mathbf{r}^{\prime} t^{\prime}\right) E_{\mu}^{(+)}(\mathbf{r} t)\right| i\right\rangle,
$$

if both photons are required to be polarized along the $\mu$ axis. The total rate at which such transitions occur is proportional to

$$
\begin{aligned}
& \sum_{f}\left|\left\langle f\left|E_{\mu}^{(+)}\left(\mathbf{r}^{\prime} t^{\prime}\right) E_{\mu}^{(+)}(\mathbf{r} t)\right| i\right\rangle\right|^{2} \\
& \quad=\left\langle i\left|E_{\mu}{ }^{(-)}(\mathbf{r} t) E_{\mu}^{(-)}\left(\mathbf{r}^{\prime} t^{\prime}\right) E_{\mu}{ }^{(+)}\left(\mathbf{r}^{\prime} t^{\prime}\right) E_{\mu}{ }^{(+)}(\mathbf{r} t)\right| i\right\rangle .
\end{aligned}
$$

Such a total rate is to be interpreted as a probability per unit (time) ${ }^{2}$ that one photon is recorded at $\mathbf{r}$ at time $t$ and another at $\mathbf{r}^{\prime}$ at time $t^{\prime}$. Photon correlation experiments of essentially the type we are describing were performed by Hanbury Brown and Twiss ${ }^{1}$ in 1955 and have, subsequently, been performed by others. ${ }^{2}$

Whatever may be the practical difficulties of more elaborate experiments, we may at least imagine the possibility of detecting $n$-fold delayed coincidences of photons for arbitrary $n$. The total rate per unit (time) ${ }^{n}$ for such coincidences will be proportional to

$$
\begin{array}{r}
\left\langle i\left|E_{\mu}{ }^{(-)}\left(\mathbf{r}_{1} t_{1}\right) \cdots E_{\mu}{ }^{(-)}\left(\mathbf{r}_{n} t_{n}\right) E_{\mu}{ }^{(+)}\left(\mathbf{r}_{n} t_{n}\right) \cdots E_{\mu}{ }^{(+)}\left(\mathbf{r}_{1} t_{1}\right)\right| i\right\rangle, \\
n=1,2,3, \cdots .
\end{array}
$$

The entire succession of such expectation values, therefore, possesses a simple physical interpretation.

In closing this survey we add a note on the commuta- 
tion rules obeyed by the fields $\mathbf{E}^{(+)}$and $\mathbf{E}^{(-)}$. It is easy to find these rules from the relation (2.3) for the real field $\mathbf{E}$, by decomposing its dependence on the two variables $t$ and $t^{\prime}$ into positive and negative frequency parts. If the function $D_{\mu \nu}$ has the Fourier transform

$$
D_{\mu \nu}\left(\mathbf{r}-\mathbf{r}^{\prime}, t-t^{\prime}\right)=\int_{-\infty}^{\infty} D_{\mu \nu}\left(\omega, \mathbf{r}-\mathbf{r}^{\prime}\right) e^{-i \omega\left(t-t^{\prime}\right)} d \omega,
$$

we see immediately that the commutator (2.3) has no part which is of positive frequency in both its $t$ and $t^{\prime}$ dependences. Neither does it have any part of negative frequency in both its time dependences. It follows that all values of the field $\mathbf{E}^{(+)}(\mathbf{r} t)$ commute with one another. and so too do those of $\mathbf{E}^{(-)}(\mathbf{r} t)$, i.e., we have

$$
\begin{aligned}
& {\left[E_{\mu}{ }^{(+)}(\mathbf{r} t), E_{\nu}{ }^{(+)}\left(\mathbf{r}^{\prime} t^{\prime}\right)\right]=0,} \\
& {\left[E_{\mu}{ }^{(-)}(\mathbf{r} t), E_{\nu}{ }^{(-)}\left(\mathbf{r}^{\prime} t^{\prime}\right)\right]=0,}
\end{aligned}
$$

for all points $\mathbf{r} t$ and $\mathbf{r}^{\prime} t^{\prime}$, and all $\mu$ and $\nu$. Products of the $\mathbf{E}^{(+)}$operators or products of the $\mathbf{E}^{(-)}$operators such as occur in (2.18) may, therefore, be freely rearranged, but the operators $\mathbf{E}^{(+)}$and $\mathbf{E}^{(-)}$do not, in general, commute.

\section{FIELD CORRELATIONS}

The electromagnetic field may be regarded as a dynamical system with an infinite number of degrees of freedom. Our knowledge of the condition of such a system is virtually never so complete or so precise in practice as to justify the use of a particular quantum state |\rangle in its description. In the most accurate preparation of the state of a field which we can actually accomplish some parameters, usually an indefinitely large number of them, must be regarded as random variables. Since there is no possibility in practice of controlling these parameters, we can only hope ultimately to compare with experiment quantities which are averages over the distributions of the unknown parameters.

Our actual knowledge of the state of the field is specified fully by means of a density operator $\rho$ which is constructed as an average, over the uncontrollable parameters, of an expression bilinear in the state vector. If |\rangle is a precisely defined state of the field corresponding to a particular set of random parameters, the density operator is defined as the averaged outer product of state vectors

$$
\rho=\{|\rangle\langle|\}_{\mathrm{av}} .
$$

The weightings to be used in the averaging are the ones which best describe the actual preparation of the fields. It is clear from the definition that $\rho$ is Hermitian, $\rho^{\dagger}=\rho$.

The average of an observable $\mathcal{O}$ in the quantum state |\rangle is the expectation value, $\langle|\mathcal{O}|\rangle$. It is the average of this quantity over the randomly prepared states which we compare with experiment. The average taken in this twofold sense may be written as

$$
\{\langle|\mathcal{O}|\rangle\}_{\mathrm{av}}=\operatorname{tr}\{\rho \mathcal{O}\}
$$

where the symbol tr stands for the trace, or the sum of the diagonal matrix elements. Since we require the average of the unit operator to be one, we must have $\operatorname{tr} \rho=1$. These considerations show that the average counting rate of an ideal photodetector, which is proportional to (2.15) in a completely specified quantum state of the field, is more generally proportional to

$$
\operatorname{tr}\left\{\rho E_{\mu}{ }^{(-)}(\mathbf{r} t) E_{\mu}{ }^{(+)}(\mathbf{r} t)\right\}
$$

when the state is less completely specified.

It is convenient at this point, as a simplification of notation, to confine our attention to a single vector component of the electric field. We suppose, for the present, that all of our detectors are fitted with polarizers and record only photons polarized parallel to an arbitrary unit vector $\mathbf{e}$. (If $\mathbf{e}$ is chosen as a complex unit vector, $\mathbf{e}^{*} \cdot \mathbf{e}=1$, the photons detected may have arbitrary elliptical polarization.) We then introduce the symbols $E^{(+)}$and $E^{(-)}$for the projections of the complex fields in the direction $\mathbf{e}$ and $\mathbf{e}^{*}$,

$$
\begin{aligned}
& E^{(+)}(\mathbf{r} t)=\mathbf{e}^{*} \cdot \mathbf{E}^{(+)}(\mathbf{r} t) \\
& E^{(-)}(\mathbf{r} t)=\mathbf{e} \cdot \mathbf{E}^{(-)}(\mathbf{r} t) .
\end{aligned}
$$

We resume a fully general treatment of photon polarizations in Sec. V.

The field average (3.3) which determines the counting rate of an ideal photodetector is a particular form of a more general type of expression whose properties are of considerable interest. In the more general expression, the fields $E^{(-)}$and $E^{(+)}$are evaluated at different spacetime points. Statistical averages of the latter type furnish a measure of the correlations of the complex fields at separated positions and times. We shall define such a correlation function, $G^{(1)}$, for the e components of the complex fields as

$$
G^{(1)}\left(\mathbf{r} t, \mathbf{r}^{\prime} t^{\prime}\right)=\operatorname{tr}\left\{\rho E^{(-)}(\mathbf{r} t) E^{(+)}\left(\mathbf{r}^{\prime} t^{\prime}\right)\right\} .
$$

Only the values of this function at $\mathbf{r}=\mathbf{r}^{\prime}$ and $t=t^{\prime}$ are needed to predict the counting rate of an ideal photodetector. However, other values of the function become necessary, quite generally, when we use as detectors less ideal systems such as actual photo-ionizable atoms. In actual photodetectors the absorption of photons can not be localized too closely, either in space or in time. Atomic photo ionization rates must be written, in general, as double integrals, over a microscopic range, of all the variables in $G^{(1)}\left(\mathbf{r} t, \mathbf{r}^{\prime} t^{\prime}\right)$. Our interest in the function $G^{(1)}$ extends to widely spaced values of its variables as well. That field correlations may extend over considerable intervals of distance and time is essential to the idea of coherence, which we shall shortly discuss.

As we have noted earlier, our interest in averages of the field operators extends beyond quadratic ones. Just as we generalized the expression for the photon detection rate to define $G^{(1)}$, we may generalize the expression 
(2.17) for the photon coincidence rate and thereby define a second-order correlation function,

$$
\begin{aligned}
& G^{(2)}\left(\mathbf{r}_{1} t_{1} \mathbf{r}_{2} t_{2}, \mathbf{r}_{3} t_{3} \mathbf{r}_{4} t_{4}\right) \\
& \quad=\operatorname{tr}\left\{\rho E^{(-)}\left(\mathbf{r}_{1} t_{1}\right) E^{(-)}\left(\mathbf{r}_{2} t_{2}\right) E^{(+)}\left(\mathbf{r}_{3} t_{3}\right) E^{(+)}\left(\mathbf{r}_{4} t_{4}\right)\right\} .
\end{aligned}
$$

This too is a function whose values, even at widely separated arguments, interest us.

In view of the possibility of discussing $n$-photon coincidence experiments for arbitrary $n$ it is natural to define an infinite succession of correlation functions $G^{(n)}$. It is convenient in writing these to abbreviate a set of coordinates $\left(\mathbf{r}_{j}, t_{j}\right)$ by a single symbol, $x_{j}$. We then define the $n$ th-order correlation function as

$$
\begin{aligned}
& G^{(n)}\left(x_{1} \cdots x_{n}, x_{n+1} \cdots x_{2 n}\right) \\
& =\operatorname{tr}\left\{\rho E^{(-)}\left(x_{1} \cdots E^{(-)} x_{n}\right) E^{(+)}\left(x_{n+1}\right) \cdots E^{(+)}\left(x_{2 n}\right)\right\} .
\end{aligned}
$$

The correlation functions have a number of simple properties. It is easily verified that interchanging the arguments in $G^{(1)}$ leads to the complex conjugate function

$$
G^{(1)}\left(\mathbf{r}^{\prime} t^{\prime}, \mathbf{r} t\right)=\left\{G^{(1)}\left(\mathbf{r} t, \mathbf{r}^{\prime} t^{\prime}\right)\right\}^{*}
$$

The same type of relation holds for all of the higher order functions

$$
G^{(n)}\left(x_{2 n} \cdots x_{1}\right)=\left\{G^{(n)}\left(x_{1} \cdots x_{2 n}\right)\right\}^{*} .
$$

Furthermore, the commutation relations (2.20) and (2.21) show us that $G^{(n)}$ is unchanged by any permutation of its arguments $\left(x_{1} \cdots x_{n}\right)$, or its arguments $\left(x_{n+1} \cdots x_{2 n}\right)$. The fact that the complex fields $E^{( \pm)}$ individually satisfy the wave equation (2.2) leads to another useful property of the $G^{(n)}$. The $n$ th-order function satisfies $2 n$ different wave equations, one for each of its arguments $x_{j},(j=1, \cdots, 2 n)$.

A large number of inequalities satisfied by the functions $G^{(n)}$ may be derived from the positive definite character of the density operator $\rho$. Derivations of several classes of these are presented in the Appendix. We confine ourselves, in this section, to mentioning some of the simpler and more useful inequalities, those which are linear or quadratic in the correlation functions. It is clear from (3.10) that all of the functions $G^{(n)}\left(x_{1} \cdots x_{n}, x_{n} \cdots x_{1}\right)$ are real. The linear inequalities assert that these functions are positive definite as well. We have then, in particular for $n=1$, the self-evident relation

and for arbitrary $n$

$$
G^{(1)}\left(x_{1}, x_{1}\right) \geq 0
$$

$$
G^{(n)}\left(x_{1} \cdots x_{n}, x_{n} \cdots x_{1}\right) \geq 0 .
$$

These relations simply affirm that the average photon intensity of a field and the average coincidence counting rates are all intrinsically positive. form

The simplest of the quadratic inequalities takes the

$$
G^{(1)}\left(x_{1}, x_{1}\right) G^{(1)}\left(x_{2}, x_{2}\right) \geq\left|G^{(1)}\left(x_{1}, x_{2}\right)\right|^{2} .
$$

Higher order inequalities of this type are given by

$$
\begin{aligned}
G^{(n)}\left(x_{1} \cdots x_{n}, x_{n} \cdots x_{1}\right) G^{(n)}\left(x_{n+1} \cdots x_{2 n}, x_{2 n} \cdots x_{n+1}\right) \\
\geq\left|G^{(n)}\left(x_{1} \cdots x_{n}, x_{n+1} \cdots x_{2 n}\right)\right|^{2},
\end{aligned}
$$

which holds for arbitrary $n$. Different forms of these relations are obtained by permuting or equating coordinates. Various other inequalities are proved in the Appendix along with those noted.

It is interesting to note that when the number of quanta present in the field is bounded, the sequence of functions $G^{(n)}$ terminates. If the density operator restricts the number of photons present to be smaller than or equal to some value $M$, the properties of $E^{( \pm)}$as annihilation and creation operators show that $G^{(n)}=0$ for $n>M$.

Classical correlation functions bearing some analogy to $G^{(1)}$ have received a great deal of discussion in recent years, mainly in connection with the theory of noise in radio waves. A detailed application of the classical correlation theory to optics has been made by Wolf. ${ }^{5}$ At the core of Wolf's analysis is a single correlation function $\Gamma$, defined as an average over an infinite time span of the product of two fields, evaluated at times separated by a fixed interval. The procedure of time averaging restricts the application of such an approach to the treatment of field distributions which are statistically stationary in time.

If we were to restrict the character of our density operator $\rho$ to describe only stationary field distributions (e.g., by choosing $\rho$ to commute with the field Hamiltonian) our function $G^{(1)}\left(\mathbf{r} t, \mathbf{r}^{\prime} t^{\prime}\right)$ would depend only on the difference of the two times, $t-t^{\prime}$. In that case the function $G^{(1)}$ would, in the classical limit (strong, lowfrequency fields), agree numerically ${ }^{6}$ with Wolf's function $\Gamma$. It should be clear, however, that the concepts of correlation and ultimately of coherence are quite useful in the discussion of nonstationary field distributions. The correlation functions $G^{(n)}$ which we have defined are ensemble averages rather than time averages and hence remain well-defined in fields of arbitrary time dependence.

\section{COHERENCE}

The term "coherence" has had long if somewhat varied use in areas of physics concerned with the electromagnetic field. In physical optics the term is used to denote a tendency of two values of the field at distantly separated points or at greatly separated times to take on correlated values. When optical means are used to superpose the fields at such points (e.g., as in Young's two-slit experiment) intensity fringes result. The possibility of producing such fringes in hypothetical superposition experiments epitomizes the optical definition of

${ }^{5}$ M. Born and E. Wolf, Principles of Optics (Pergamon Press, Inc., London, 1959), Chap. X. An extensive bibliography is given there.

"This is true provided Wolf's "disturbance" field $V$ behaves ergodically and is identified with $E^{(+)}$. 
coherence. The definition has remained a satisfactorily explicit one only as long as optical experiments were confined to measuring field intensities, or more generally quantities quadratic in the field strengths. We have already noted that the photon correlation experiment of Hanbury Brown and Twiss, ${ }^{1}$ performed in 1955, is of an altogether new type and measures the average of a quartic expression. ${ }^{7}$ The study of quantities of fourth and higher powers in the field strengths is the basis of all work in the recently developed area of nonlinear optics. It appears safe to assume that the number of such experiments will increase in the future, and that the concept of coherence should be extended to apply to them.

Another pressing reason for sharpening the meaning of coherence is provided by the recent development of the optical maser. The maser produces light beams of narrow spectral bandwidth which are characterized by field correlations extending over quite long ranges. Such light is inevitably described as coherent, but the sense in which the term is used has not been made adequately clear. If the sense is simply the optical one then, as we shall see, it may scarcely do justice to the potentialities of the device. The optical definition does not at all distinguish among the many ways in which fields may vary while remaining equally correlated at all pairs of points. That much greater regularities may exist in the field variations of a maser beam than are required by the optical definition of coherence may be seen by comparing the maser beam with the carrier wave of a radio transmitter. The latter type of wave ideally possesses a stability of amplitude which optically coherent fields need not have. ${ }^{8}$ Furthermore, the field values of such a wave possess correlations of a much more detailed sort than the optical definition requires. These are properties best expressed in terms of the higher order correlation functions $G^{(n)}$, for $n>1$.

To discuss coherence in quantitative terms it is convenient to introduce normalized forms of the correlation functions. Corresponding to the first-order function $G^{(1)}$ we define

$$
g^{(1)}\left(\mathbf{r} t, \mathbf{r}^{\prime} t^{\prime}\right)=\frac{G^{(1)}\left(\mathbf{r} t, \mathbf{r}^{\prime} t^{\prime}\right)}{\left\{G^{(1)}(\mathbf{r} t, \mathbf{r} t) G^{(1)}\left(\mathbf{r}^{\prime} t^{\prime}, \mathbf{r}^{\prime} t^{\prime}\right)\right\}^{1 / 2}} .
$$

It is immediately seen from (3.13) that $g^{(1)}$ obeys the inequality

$$
\left|g^{(1)}\left(\mathbf{r} t, \mathbf{r}^{\prime} t^{\prime}\right)\right| \leq 1
$$

For $\mathbf{r}=\mathbf{r}^{\prime}, t=t^{\prime}$ we have, of course, $g^{(1)} \equiv 1$.

The normalized forms of the higher order correlation functions are defined as

$g^{(n)}\left(x_{1} \cdots x_{2 n}\right)=G^{(n)}\left(x_{1} \cdots x_{2 n}\right) / \prod_{j=1}^{2 n}\left\{G^{(1)}\left(x_{j}, x_{j}\right)\right\}^{1 / 2}$.

${ }^{7}$ R. J. Glauber, Phys. Rev. Letters 10, 84 (1963). The particular field referred to as incoherent in that note may have first-order coherence if it is monochromatic, but not second- or higher order coherence.

${ }^{8}$ This point has been noted with particular clarity by M. J. E. Golay, Proc. IRE 49, 959 (1961); also 50, 223 (1962).
These functions, for $n>1$, are not, in general, restricted in absolute value as is $g^{(1)}$.

We shall try in this paper to give the concept of coherence as precise a definition as is both realizable in physical terms, and useful as well. ${ }^{9} \mathrm{We}$, therefore, begin by stating an infinite sequence of conditions on the functions $g^{(n)}$ which are to be satisfied by a fully coherent field. These necessary conditions for coherence are that the normalized correlation functions all have unit absolute magnitude,

$$
\left|g^{(n)}\left(x_{1} \cdots x_{2 n}\right)\right|=1, \quad n=1,2 \cdots .
$$

That there exist at least some states which meet these conditions at all points of space and time is immediately clear from the example of a classical plane wave, $E^{(+)} \sim \exp [i(\mathbf{k} \cdot \mathbf{r}-\omega t)]$. We shall presently show that the class of coherent fields is vastly larger than that of individual plane waves.

The conditions (4.4) on the functions $g^{(n)}$ are stated only as necessary ones and need not be construed as defining coherence completely. We shall shortly, in fact, sharpen the definition somewhat further. It is worth noting at this point, however, that not all of the fields which have been described as "coherent" in the past meet the set of conditions (4.4) even approximately. There may be some virtue, therefore, in constructing a hierarchy of orders of coherence to discuss fields which do not have that property in its fullest sense. We shall state as a condition necessary for first-order coherence that $\left|g^{(1)}\left(\mathbf{r} t, \mathbf{r}^{\prime} t^{\prime}\right)\right|=1$. More generally, for a field to be characterized by $n$th order coherence we shall require $\left|g^{(j)}\right|=1$ for $j \leq n$. For fields which occur in practice, one can not expect relations such as these to hold exactly for all points in space and time. We shall, therefore, often employ the term $n$th order coherence more loosely to mean that the first $n$ coherence conditions are fairly accurately satisfied over appreciable intervals of the variables surrounding all points $x_{1}=x_{2}=\cdots=x_{2 n}$.

The definition of coherence which has been used to date in all studies of physical optics corresponds only to first-order coherence. The most coherent fields which have been generated by optical means prior to the development of the maser, in fact, lack second and higher order coherence. On the other hand, the optical maser, functioning with ideal stability, may produce fields which are coherent to all orders.

The various orders of coherence may, in principle, be distinguished fairly directly in experimental terms. The inequality (3.12), which states that the $n$-fold coincidence counting rate is positive, requires that $g^{(n)}\left(x_{1} \cdots x_{n}, x_{n} \cdots x_{1}\right)$ be positive. If the field in question possesses $n$ th-order coherence, it must, therefore, have

$$
g^{(j)}\left(x_{1} \cdots x_{j}, x_{j} \cdots x_{1}\right)=1,
$$

${ }^{9} \mathrm{~A}$ brief account of this work was presented by R. J. Glauber, in Proceedings of the Third International Conference on Quantum Electronics, Paris, France, 1963 (to be published). 
for $j \leq n$. It follows from the definitions of the $g^{(j)}$ that the corresponding values of the correlation functions $G^{(j)}$ factorize, i.e.,

$$
G^{(j)}\left(x_{1} \cdots x_{j}, x_{j} \cdots x_{1}\right)=\prod_{i=1}^{j} G^{(1)}\left(x_{i}, x_{i}\right),
$$

for $j \leq n$. These relations mean, in observational terms, that the rate at which $j$-fold delayed coincidences are detected by our ideal photon counters, reduces to a product of the detection rates of the individual counters. ${ }^{7}$ In photon coincidence experiments of multiplicity up to and including $n$, the photon counts registered by the individual counters may then be regarded as statistically independent events. No tendency of photon counts to be statistically correlated will be evident in $j$-fold coincidence experiments for $j \leq n$.

The experiments of Hanbury Brown and Twiss ${ }^{1}$ were designed to detect correlations in the fluctuating outputs of two photomultipliers. These detectors were placed in fields made coherent with one another (in the optical sense) through the use of monochromatic, pinhole illumination and a semitransparent mirror. The photocurrents of the two detectors were observed to show a positive correlation for small delay times, rather than independent fluctuations. A similar experiment has been performed by Rebka and Pound, ${ }^{2}$ using coincidence counting equipment. Their experiment, performed with a more monochromatic beam and better geometrical definition, shows an explicit correlation in the counting probabilities of the two detectors. These observations verify that light beams from ordinary sources such as discharge tubes, when made optimally coherent in the first-order sense, still lack second-order coherence.

The coherence conditions (4.4) can also be stated as a requirement that the functions $\left|G^{(n)}\left(x_{1} \cdots x_{2 n}\right)\right|$ factorize into a product of $2 n$ functions of the same form, each dependent on a single space-time variable,

$$
\left|G^{(n)}\left(x_{1} \cdots x_{2 n}\right)\right|=\prod_{j=1}^{2 n}\left\{G^{(1)}\left(x_{j}, x_{j}\right)\right\}^{1 / 2} .
$$

This statement of the necessary conditions for coherence suggests that it may be convenient to give a stronger definition to coherence by regarding it as a factorization property of the correlation functions,

Let us suppose that there exists a function $\mathcal{E}(x)$, independent of $n$, such that the correlation functions for all $n$ may be expressed as the products

$$
\begin{aligned}
& G^{(n)}\left(x_{1} \cdots x_{n}, x_{n+1} \cdots x_{2 n}\right) \\
&=\mathcal{E}^{*}\left(x_{1}\right) \cdots \mathcal{E}^{*}\left(x_{n}\right) \mathcal{E}\left(x_{n+1}\right) \cdots \mathcal{E}\left(x_{2 n}\right) .
\end{aligned}
$$

It is immediately clear that these functions satisfy the conditions (4.4) and (4.7). To show that fields with such correlations exist we need only refer again to the case of a classical plane wave. In fact, any classical field of predetermined (i.e., nonrandom) behavior has correlation functions which fall into this form, and such fields are at times called coherent in communication theory. We shall, therefore, adopt the factorization conditions (4.8) as the definition of a coherent field and turn next to the question of how they may be satisfied in the quantum domain.

If it were possible for the field to be in an eigenstate of the operators $E^{(+)}$and $E^{(-)}$, the correlation functions for such states would factorize immediately to the desired form. The operators $E^{(+)}(\mathbf{r} t)$ and $E^{(-)}\left(\mathbf{r}^{\prime} t^{\prime}\right)$ do not commute, however, so no state can be an eigenstate of both in the usual sense. Not only are these operators nonHermitian, but the failure of each to commute with its adjoint shows that $E^{(+)}$and $E^{(-)}$are non-normal as well. Operators of this type can not, as a rule, be diagonalized at all, but may nonetheless have eigenstates. In general, we must distinguish between their left and right eigenstates; the two types need not occur in mutually adjoint pairs. The operator $E^{(+)}(\mathbf{r} t)$, in particular, has no left eigenstates, but does have right eigenstates ${ }^{10}$ corresponding to complex eigenvalues for the field, which are functions of position and time. We shall suppose that |\rangle is a right eigenstate of $E^{(+)}$and that the equation it satisfies takes the form

$$
E^{(+)}(\mathbf{r} t)|\rangle=\mathscr{E}(\mathbf{r} t)|\rangle
$$

in which the function $\mathcal{E}(\mathbf{r} t)$ is to be interpreted as the complex eigenvalue. The Hermitian adjoint of this relation shows us that the conjugate state, $\langle l$, is a left eigenstate of $E^{(-)}(\mathbf{r} t)$,

$$
\langle| E^{(-)}(\mathbf{r} t)=\langle| \mathcal{E}^{*}(\mathbf{r} t) .
$$

The density operator for such states is simply the projection operator, $\rho=|\rangle\langle|$. It follows immediately from these relations that the correlation functions $G^{(n)}$ all factorize into the form of Eq. (4.8). In other words, the state of the field defined by Eqs. (4.9) or (4.10) meets our definition precisely and is fully coherent. We shall discuss the properties of such states ${ }^{11}$ at length in the paper to follow. For the present it may suffice to say that we can find an eigenstate |\rangle which corresponds to the choice, as an eigenvalue, of any function $\mathcal{E}(\mathbf{r} t)$ which satisfies certain conditions. One condition, which is clear from Eq. (4.9), is that $\mathcal{E}(\mathbf{r} t)$ must satisfy the wave equation. The other, which corresponds to the positive frequency character of $E^{(+)}$, is that $\mathcal{E}(\mathbf{r} t)$, when regarded as a function of a complex time variable, be analytic in the lower half-plane. The eigenstates which correspond to different fields $\mathcal{E}(\mathbf{r} t)$ are not mutually orthogonal, but nontheless form a natural basis for the discussion of photon detection problems. We have introduced them

\footnotetext{
${ }^{10}$ States of the harmonic oscillator which have an analogous property were introduced in a slightly different but related connection by E. Schrödinger, Naturwiss. 14, 664 (1926). The electromagnetic field, as is well known, may be treated as an assembly of oscillators.

1 Some of the properties of these states have already been noted in references 7 and 9 .
} 
here only to demonstrate the possibility of satisfying the coherence conditions in quantum theory. Such quantum states do not exhaust the possibility of describing coherent fields. Statistical mixtures, for example, of the states for which the eigenvalues $\mathcal{E}(\mathbf{r} t)$ differ by constant phase factors satisfy the coherence conditions equally well.

The fields which have been described as most coherent in optical contexts have tended to be those of the narrowest spectral bandwidth. If coherent fields in optics have necessarily been chosen as monochromatic ones, it is because that has been virtually the only means of securing appreciably correlated fields from intrinsically chaotic sources. For this reason, perhaps, there has been a natural tendency to associate the concept of coherence with monochromaticity. The association was, in fact, made an implicitly rigid one by earlier discussions $^{5}$ of optical (i.e., first-order) coherence which were applicable only to statistically stationary fields. By extending the definition of coherence to nonstationary fields we see that it places no constraint on the frequency spectrum. Coherent fields exist corresponding to eigenvalues $\mathcal{E}(\mathbf{r} t)$ with arbitrary spectra. The coherence conditions restrict randomness of the fields rather than their bandwidth.

Having defined full coherence by means of the factorization conditions (4.8), we may now use them in defining the various orders of coherence. We shall speak of $m$ th-order coherent fields when the conditions (4.8) are satisfied for $n \leq m$, a definition which accords with our earlier conditions on $\left|g^{(j)}\right|$.

Photon correlation experiments have shown the importance of distinguishing between the first two orders of coherence. At the other end of the scale, we have shown that there exist, in principle at least, states which are fully coherent. We are entitled to ask, therefore, whether the intermediate orders of coherence will also be useful classifications. In the absence of any experimental information, we can only guess that they may be useful, though perhaps not in the sharp sense in which we we have defined them. One may easily imagine the possibility that, for light sources such as the maser, the correlation functions $G^{(n)}$ show gradually increasing departure from the factored forms (4.8) as $n$ increases, even when the variables $x_{1} \cdots x_{2 n}$ are not too widely separated. In such contexts the order of coherence can only be defined approximately. ${ }^{12}$ Something of the same approximate character must be present in all applications of the definitions we have given. The field correlations we have discussed can extend over great intervals

12 The characterization we have given the $n$ th-order coherent fields is, in principle, an accurately realizable one, however. States with such properties may be constructed in a variety of ways. The factorization conditions can be met for $j \leq n$, for example, by suitably chosen states in which the number of photons present may take on any value up to $n$. The correlation functions of order $j>n$ then vanish, as we have noted earlier. The vanishing of these correlation functions for states with bounded numbers of quanta shows, incidentally, that no bound can be placed on the photon number in a fully coherent field. of distance and time, though never infinite ones in practice. Coherence conditions, such as $\left|g^{(n)}\right|=1$, can only be met within a finite range of relative values of the coordinates $x_{1} \cdots x_{2 n}$. It is only within such ranges, and therefore as an approximation, that we can speak of coherence at all.

\section{COHERENCE AND POLARIZATION}

We have to this point, in the interest of simplicity, dealt only with the projections of the fields along a single (possibly complex) unit vector e. To take fuller account of the vector nature of the fields we must define tensor rather than scalar correlation functions. The first-order function is taken to be

$$
G_{\mu \nu}{ }^{(1)}\left(x, x^{\prime}\right)=\operatorname{tr}\left\{\rho E_{\mu}^{(-)}(x) E_{\nu}^{(+)}\left(x^{\prime}\right)\right\},
$$

in which the indices $\mu$ and $\nu$ label Cartesian components. This function satisfies the symmetry relation

$$
G_{\nu \mu}^{(1)}\left(x^{\prime}, x\right)=\left\{G_{\mu \nu}{ }^{(1)}\left(x, x^{\prime}\right)\right\}^{*},
$$

and is shown in the appendix to obey the inequalities,

and

$$
G_{\mu \mu}^{(1)}(x, x) \geq 0
$$

$$
G_{\mu \mu}{ }^{(1)}(x, x) G_{\nu \nu}{ }^{(1)}\left(x^{\prime}, x^{\prime}\right) \geq\left|G_{\mu \nu}{ }^{(1)}\left(x, x^{\prime}\right)\right|^{2} .
$$

The photon intensities which can be detected at the space-time point $x$ are found from $G_{\mu \nu}{ }^{(1)}\left(x, x^{\prime}\right)$ for $x^{\prime}=x$. We shall abbreviate this $3 \times 3$ matrix as $\mathbf{G}^{(1)}(x)$, and use it as the basis of a brief discussion of polarization correlations in three dimensions, a subject which seems to have received little attention in comparison to plane polarizations. The symmetry relation (5.2) for $x^{\prime}=x$ shows that the intensity matrix $\mathbf{G}^{(1)}(x)$ is Hermitian; an argument given in the Appendix shows it to be a positive definite matrix as well. It follows that $\mathbf{G}^{(1)}(x)$ has positive real eigenvalues, $\lambda_{p}(x),(p=1,2,3)$, which correspond to a set of (generally, complex) eigenvectors. The eigenvectors, which we write as $\mathbf{e}^{(p)}$ satisfy

$$
\begin{aligned}
\mathbf{G}^{(1)}(x) \cdot \mathbf{e}^{(p) *} & =\lambda_{p} \mathbf{e}^{(p) *}, \\
\mathbf{e}^{(p)} \cdot \mathbf{G}^{(1)}(x) & =\lambda_{p} \mathbf{e}^{(p)} .
\end{aligned}
$$

If the three eigenvalues $\lambda_{p}(x)$ are all different, it is clear that the three eigenvectors must be orthogonal; if not they may be chosen so. If the eigenvectors are normalized to obey the relations

$$
\mathbf{e}^{(p)} \cdot \mathbf{e}^{(q) *}=\delta_{p q},
$$

their components form the unitary matrix which diagonalizes $\mathbf{G}^{(1)}(x)$. The eigenvectors, or equivalently the unitary matrix, are determined by a set of eight independent real parameters.

A tensor product, such as

$$
\mathbf{e}^{(p)} \cdot \mathbf{G}^{(1)}(x) \cdot \mathbf{e}^{(q) *}=\lambda_{p} \delta_{p q},
$$

expresses the correlation, at the point $x$, of the field components in the $\mathbf{e}^{(p)}$ and $\mathbf{e}^{(q)}$ directions. It is clear, 
then, that there always exist a set of three (complex) orthogonal polarization vectors such that the field components in these directions are statistically uncorrelated. The eigenvalues $\lambda_{p}$ correspond to the intensities for these polarizations. For quantitative discussions of polarization it is convenient to define the normalized intensities $I_{p}=\lambda_{p} / \sum_{q} \lambda_{q},(p=1,2,3)$, which sum to unity, $\sum_{p} I_{p}=1$. When the normalized intensities are all equal to $\frac{1}{3}$ we have the case of an isotropic field, as in a hohlraum filled with thermal radiation.

The triad of eigenvectors at a point in an arbitrary field depends, in general, on time as well as position. If the density operator, $\rho$, represents a stationary ensemble, however, the triad becomes fixed. A particular example which has been studied in minute detail in optics is that of a beam of plane waves. ${ }^{5,13}$ In that case, since the fields are transverse, one of the eigenvectors may be chosen as the beam direction and obviously corresponds to the eigenvalue zero. The net polarization of the beam is usually defined as the magnitude of the difference of the normalized intensities, $\left|I_{1}-I_{2}\right|$, which correspond to the remaining two eigenvalues.

We next define the higher order correlation functions as

$$
\begin{array}{r}
G_{\mu_{1} \cdots \mu_{2 n}}\left(x_{1} \cdots x_{n}, x_{n+1} \cdots x_{2 n}\right)=\operatorname{tr}\left\{\rho E_{\mu_{1}}{ }^{(-)}\left(x_{1}\right) \cdots\right. \\
\left.\times E_{\mu_{n}}{ }^{(-)}\left(x_{n}\right) E_{\mu_{n+1}}{ }^{(+)}\left(x_{n+1}\right) \cdots E_{\mu_{2 n}}{ }^{(+)}\left(x_{2 n}\right)\right\} .
\end{array}
$$

These functions are unchanged by simultaneous permutations of the coordinates $\left(x_{1} \cdots x_{n}\right)$ and the indices $\left(\mu_{1} \cdots \mu_{n}\right)$; they are likewise invariant under permutations of the $\left(x_{n+1} \cdots x_{2 n}\right)$ and $\left(\mu_{n+1} \cdots \mu_{2 n}\right)$. They satisfy the symmetry relation

$$
G_{\mu_{2 n} \cdots \mu_{1}}^{(n)}\left(x_{2 n} \cdots x_{1}\right)=\left\{G_{\mu_{1} \cdots \mu_{2 n}}^{(n)}\left(x_{1} \cdots x_{2 n}\right)\right\}^{*}
$$

and are shown, in the Appendix, to obey the inequalities

and

$$
G_{\mu_{1} \cdots \mu_{n} \mu_{n} \cdots \mu_{1}}^{(n)}\left(x_{1} \cdots x_{n}, x_{n} \cdots x_{1}\right) \geq 0
$$

$$
\begin{aligned}
& G_{{ }_{\mu 1} \cdots \mu_{n} \mu_{n} \cdots \mu_{1}}^{(n)}\left(x_{1} \cdots x_{n}, x_{n} \cdots x_{1}\right) \\
& \quad \times G_{\mu_{n+1}^{(n)} \cdots \mu_{2 n} \mu_{2 n} \cdots \mu_{n+1}}\left(x_{n+1} \cdots x_{2 n}, x_{2 n} \cdots x_{n+1}\right) \\
& \quad \geq\left|G_{\mu_{1} \cdots \mu_{n} \mu_{n+1} \cdots \mu_{2 n}}^{(n)}\left(x_{1} \cdots x_{n}, x_{n+1} \cdots x_{2 n}\right)\right|^{2} .
\end{aligned}
$$

As in our earlier discussion of coherence, it is convenient to make use of the normalized correlation functions

$$
\begin{aligned}
& g_{\mu_{1} \cdots \mu_{2 n}}^{(n)}\left(x_{1} \cdots x_{2 n}\right) \\
& \quad=G_{\mu_{1} \cdots \mu_{2 n}}^{(n)}\left(x_{1} \cdots x_{2 n}\right) / \prod_{j=1}^{2 n}\left\{G_{\mu_{j} \mu_{j}}^{(1)}\left(x_{j}, x_{j}\right)\right\}^{1 / 2} .
\end{aligned}
$$

The necessary conditions for full coherence are

$$
\left|g_{\mu_{1} \cdots \mu_{2 n}}^{(n)}\left(x_{1} \cdots x_{2 n}\right)\right|=1 \text {, }
$$

which must hold for all components $\mu_{1} \cdots \mu_{2 n}$, as well as all $n$. It is clear, however, that these conditions do not

\footnotetext{
${ }^{13}$ Most of these studies have been confined to stationary, quasimonochromatic beams. See, for example, G. B. Parrent, Jr., and P. Roman, Nuovo Cimento 15, 370 (1960).
}

constitute an adequate definition of coherence, since they are not, in general, invariant under rotations of the coordinate axes. We therefore turn once again to a definition of coherence as a factorization property of the correlation functions.

We define full coherence to hold when the set of correlation functions $G^{(n)}$ may be expressed as products of the components of a vector field $\mathcal{E}_{\mu}(x),(\mu=1,2,3)$, i.e., $G^{(n)}{ }_{\mu_{1} \cdots \mu_{2 n}}\left(x_{1} \cdots x_{n}, x_{n+1} \cdots x_{2 n}\right)$

$$
=\prod_{j=1}^{n} \mathcal{E}^{*}{ }_{\mu_{j}}\left(x_{j}\right) \prod_{l=n+1}^{2 n} \mathcal{E}_{\mu_{l}}\left(x_{l}\right)
$$

where it is understood that the vector field $\mathcal{E}_{\mu}(x)$ is independent of $n$. It is immediately clear, from the transformation properties of the definition, that a field coherent in one coordinate frame is equally coherent in any rotated frame. Furthermore, all of the normalized correlation functions $g^{(n)}$, which follow from the definition, satisfy the conditions (5.13).

The coherence conditions (5.14) imply that the field is fully polarized in the direction of the vector $\varepsilon(x)$ at each point $x$. The formal way of seeing this is to note that the intensity matrix $G_{\mu \nu}{ }^{(1)}(x, x)$, which we discussed earlier in general terms, reduces for a coherent field to,

$$
G_{\mu \nu}{ }^{(1)}(x, x)=\mathcal{E}_{\mu}{ }^{*}(x) \mathcal{E}_{\nu}(x) .
$$

Such a matrix represents an unnormalized projection operator for the direction of $\boldsymbol{\varepsilon}(x)$. It obviously has, as an eigenvector in the sense of Eq. (5.5), the vector $\mathcal{E}_{\mu}(x)$ itself. The corresponding eigenvalue is the full intensity $\sum_{\mu}\left|\mathcal{E}_{\mu}(x)\right|^{2}$. The two remaining eigenvalues, which correspond to orthogonal directions, clearly vanish.

It is interesting to note that for coherent fields many of the inequalities stated earlier, e.g., (3.13), (3.14), (5.4), (5.11), reduce to statements of equality. This reduction holds quite generally, as is shown in the Appendix, for those inequalities of quadratic and higher degree in the correlation functions.

The arguments by which we exhibit fields satisfying the coherence conditions, are essentially unchanged from the previous section. In particular, as we shall discuss in the next paper, there exist states which are simultaneously right eigenstates of all three components of $E_{\mu}{ }^{(+)}(\mathbf{r} t)$ and correspond to a set of three complex eigenvalues $\mathcal{E}_{\mu}(\mathbf{r} t)$. Such states satisfy the coherence conditions (5.14) precisely.

If we have chosen to discuss only the correlations of the electric field in this paper, it is because that field plays the dominant role in all detection mechanisms for photons of lower frequency than $\mathrm{x}$ rays. It is not difficult to construct correlation functions which involve the magnetic field as well as the electric field, and perhaps these too will someday prove useful. One method is to use the relativistic field tensor, $F_{\mu \nu}$, in precisely the way we have used the field $E_{\mu}$. The field tensor may be written as a $4 \times 4$ antisymmetric matrix, made up of the 
components of both the electric and magnetic fields. The $n$ th-order correlation function for the complex components of those fields would have $4 n$ four-valued indices. Coherence may then be defined as a requirement that the correlation functions all be separable into the the products of $4 \times 4$ antisymmetric fields, just as Eq. (5.14) requires a separation into products of three-vector field components. The advantage of such a definition is to make it clear that coherence is a relativistically invariant concept; that a field which is coherent in any one Lorentz frame is coherent in any other. Fields which are coherent in this relativistic sense are automatically coherent in the more limited senses we have described earlier.

\section{ACKNOWLEDGMENTS}

The author is grateful to the Research Laboratory of the American Optical Company and its director, Dr. S. M. MacNeille, for partial support of this work.

\section{APPENDIX}

In this section we derive a number of inequalities obeyed by the correlation functions defined in the paper. Fundamentally, these relations are all consequences of a single inequality

$$
\operatorname{tr}\left\{\rho A^{\dagger} A\right\} \geq 0
$$

which holds for arbitrary choice of the operator $A$. To prove this inequality, we note that the density operator $\rho$ is Hermitian and can always be diagonalized, i.e., we can find a set of basis states such that the matrix representation of $\rho$ is

$$
\langle k|\rho| l\rangle=\delta_{k l} p_{k}
$$

The numbers $p_{k}$ may be interpreted as probabilities associated with the states $|k\rangle$. They are, therefore, nonnegative, $p_{k} \geq 0$; which is to say that $\rho$ is a positive definite operator. The normalization condition on the density operator, $\operatorname{tr} \rho=\sum p_{k}=1$, shows that not all the $p_{k}$ vanish. The trace (A1) may be reduced, in the representation defined by (A2), to the form

$$
\operatorname{tr}\left\{\rho A^{\dagger} A\right\}=\sum_{k} p_{k}\left\langle k\left|A^{\dagger} A\right| k\right\rangle .
$$

The diagonal matrix elements on the right of (A3) are all non-negative since they may be expressed as a sum of squared absolute values,

$$
\begin{aligned}
\left\langle k\left|A^{\dagger} A\right| k\right\rangle & =\sum_{l}\left\langle k\left|A^{\dagger}\right| l\right\rangle\langle l|A| k\rangle \\
& =\sum_{l}|\langle l|A| k\rangle|^{2} .
\end{aligned}
$$

This statement completes the proof of (A1), since the trace is invariant under unitary transformations of the basis states.

The trace which occurs in the inequality (A1) has the same basic structure as all of the correlation functions $G^{(n)}$. Various inequalities relating the correlation functions follow, more or less directly, from different choices of the operator $A$. If, for example, we choose $A$ to be $E^{(+)}(x)$, as defined by (3.4), we find the inequality (3.11),

$$
G^{(1)}(x, x) \geq 0
$$

If we choose $A$ to be the $n$-fold product $E^{(+)}\left(x_{1}\right) \cdots$ $E^{(+)}\left(x_{n}\right)$ we find the inequality (3.12),

$$
G^{(n)}\left(x_{1} \cdots x_{n}, x_{n} \cdots x_{1}\right) \geq 0 .
$$

The proofs are no different if the components of the three-dimensional field are used in place of $E^{(+)}$, i.e., if a component index $\mu_{j}$ is associated with each coordinate $x_{j}$. Hence, we have also derived (5.3) and (5.10).

The remaining inequalities are of second and higher degree in the correlation functions. Those obeyed by the first-order function, $G^{(1)}$, may be found as follows: We choose at random a set of $m$ space-time points $x_{1} \cdots x_{m}$, and consider as the operator $A$,

$$
A=\sum_{j=1}^{m} \lambda_{j} E^{(+)}\left(x_{j}\right),
$$

where the superposition coefficients $\lambda_{1} \cdots \lambda_{m}$ are an arbitrary set of complex numbers. When we substitute (A7) into the basic inequality, (A1), we find

$$
\sum_{i, j} \lambda_{i}^{*} \lambda_{j} G^{(1)}\left(x_{i}, x_{j}\right) \geq 0
$$

In other words, the set of correlation functions $G^{(1)}\left(x_{i}, x_{j}\right)$ for $i, j=1, \cdots, m$ forms the matrix of coefficients of a positive definite quadratic form. It follows, in particular, that the determinant of the matrix is non-negative,

$$
\operatorname{det}\left[G^{(1)}\left(x_{i}, x_{j}\right)\right] \geq 0 \quad i, j=1, \cdots, m .
$$

For $m=1$ this inequality is simply (A5). For $m=2$ it becomes the one noted in the text as (3.13),

$$
G^{(1)}\left(x_{1}, x_{1}\right) G^{(1)}\left(x_{2}, x_{2}\right) \geq\left|G^{(1)}\left(x_{1}, x_{2}\right)\right|^{2} .
$$

For larger values of $m$ the inequalities are perhaps best left in the form (A9). When tensor components are introduced, we have only to replace the coordinate $x_{j}$ in the proofs by the combination of $x_{j}$ and a tensor index $\mu_{j}$. The relation (5.4) thereby follows from the form of (A10). If, in particular for $m=3$, we choose the three coordinates to be the same and the tensor indices all different, i.e., we choose

$$
A=\sum_{\nu=1}^{3} \lambda_{\nu} E_{\nu}{ }^{+}(x),
$$

we find that the $3 \times 3$ matrix $G_{\mu \nu}{ }^{(1)}(x, x)$ is positive definite, a property used in the text in the discussion of polarizations.

Since the succession of inequalities which follows from (A1) is endless, we only mention the quadratic ones for 
the higher order functions. To find these, we choose a set of $2 n$ coordinates at random and let $A$ be any operator of the form

$$
\begin{aligned}
A=\lambda_{1} E^{(+)}\left(x_{1}\right) \cdots E^{(+)}\left(x_{n}\right) & \\
& +\lambda_{2} E^{(+)}\left(x_{n+1}\right) \cdots E^{(+)}\left(x_{2 n}\right) .
\end{aligned}
$$

The positive definiteness of the quadratic form which results from substituting this expression in (A1) shows that the inequality (3.14) must hold. When vector indices are attached to the operators $E^{(+)}$, the same proof leads to (5.11).

We have noted in the text that, for the particular case of coherent fields, the inequalities of second degree in the correlation functions reduce to equalities. The reason for the reduction lies in the way the correlation functions factorize. The factorization causes all of the second and higher order determinants involved in the statement of positive definiteness conditions [e.g., (A9)] to vanish.

\title{
Statistical Mechanical Theory of Condensation*
}

\author{
M. Coopersmith $\dagger$ and R. Brout $\ddagger$ \\ Laboratory of Atomic aud Solid State Physics and Department of Physics, Cornell University, Ithaca, New York
}

(Received 6 July 1962; revised manuscript received 9 October 1962)

\begin{abstract}
The cluster expansion for the free energy of the Ising model is reinterpreted as a cluster expansion for the pressure of the lattice gas. It is observed that the free energy of the Ising model is a function of $1-R^{2}$, where $R$ is the long-range order, so that the pressure of the lattice gas is a function of $\rho(1-\rho)$, where $\rho$ is density. The factor $1-\rho$ comes from the prevention of more than one particle occupying a lattice site. This idea has motivated the development of a real hard-core gas with a weak attractive tail. A cluster expansion is developed in terms of the tail alone and the hard core is treated as a metric (i.e., the hard-core part of the potential is treated exactly in all integrals). Pressure-volume isotherms are calculated explicitly in the zeroth order or molecular field approximation using the Lennard-Jones (6-12) potential and good quantitative results are found for the critical parameters as well as a qualitative understanding of the condensation phenomena. The theory of the first-order correction (spherical model) is then outlined and fluctuations of the OrnsteinZernicke type in the local density are found. The theory of condensation is qualitatively understood in the sense that the Weiss field gives an understanding of ferromagnetism. The theory of the detailed fluctuations in the critical region is equally difficult for both phenomena since the problems are put on the same footing in the present paper.
\end{abstract}

\section{INTRODUCTION}

$I^{\mathrm{N}}$ $\mathrm{N}$ a series of papers, Brout and Horwitz ${ }^{1}$ have shown how to obtain a linked cluster expansion for the Ising model and, in particular, how to calculate the spherical model value for the free energy as a highdensity limit of the cluster expansion. We note the trivial but important fact that the spherical model value of the free energy below the Curie point is a function of $1-R^{2}$, where $R$ is the long-range order of magnetization. Reinterpreting the free energy of the Ising model as the pressure of a lattice gas in the manner of Lee and Yang, ${ }^{2}$ we observe that the lattice gas pres-

\footnotetext{
* Supported in part by the Office of Naval Research.

$\dagger$ This paper is abstracted from the dissertation of M. Coopersmith submitted to Cornell University in partial fulfillment for the requirements of the doctoral degree. National Science Postdoctoral Fellow 1961-1962. Present address: Institute for the Study of Metals, University of Chicago, Chicago, Illinois.

† John Simon Guggenheim Fellow 1961-1962. Present address: Université Libre de Bruxelles, Brussels, Belgium.

1 R. Brout, Phys. Rev. 115, 824 (1959); 118, 1009 (1960); 122 469 (1961); G. Horwitz and H. Callen, ibid. 124, 1757 (1961)

Hereafter referred to as I, II, III, and IV, respectively.

${ }_{2}$ T. D. Lee and C. N. Yang, Phys. Rev. 87, 410 (1952) ; 87, 404 (1952).
}

sure is a function of $\rho(1-\rho)$, where $\rho$ is the density. Since the factor $1-\rho$ comes about from the fact that no more than one particle is allowed on a lattice site (hard core), we conjecture that it might be profitable to formulate a cluster expansion for a real hard-core gas with a weak attractive tail using the tail alone as the perturbation and calculating all ensemble averages with the hard core as a metric (i.e., the cluster integrals which occur contain the hard sphere part exactly taking into account no overlapping of the cores).

In Sec. II, we review briefly the cluster expansion for the Ising model and the high-density limit (spherical model) as a sum of ring graphs (random phase approximation). In Sec. III, we present this cluster expansion by a method which uses the lattice gas interpretation of the Ising model rather than the conventional spin method. The advantage of this method is that the cluster expansion for a real gas with a hard core can be developed in a parallel manner. In Sec. IV, we show how to formulate this linked cluster expansion for an imperfect hard-core gas. Finally, in Sec. V we calculate the pressure-volume isotherms for the real gas using the zeroth order of molecular field approximation to the 